\title{
Incidence and risk factors for new onset diabetes after liver transplantation in a hospital of Buenos Aires. P-0495
}

Authors: J.N. Giunta1 , C.S. Bermudez², S. Marciano ${ }^{3}$, C.J. Rodriguez¹, M.E. Gullace ${ }^{1}$, V.L. Pagotto ${ }^{4}$, M.M. Castex $^{1}$, L.I. Capurro ${ }^{1}$, A.M. Alvarez¹, D.H. Giunta ${ }^{4}$, A.C. Gadano ${ }^{3}$, L.A. Grosembacher1.

${ }^{1}$ Hospital Italiano de Buenos Aires, Endocrinology, CABA, Argentina.

${ }^{2}$ Hospital Italiano de Buenos Aires, Hepatology, Buenos Aires, Argentina.

${ }^{3}$ Hospital Italiano de Buenos Aires, Hepatology and Department of Clinical Research, Buenos Aires, Argentina

${ }^{4}$ Hospital Italiano de Buenos Aires, Department of Clinical Research, Buenos Aires, Argentina.

\section{Background}

New onset diabetes after liver transplantation is a frequent complication related to the type of immunosuppression, frequency of rejection, recurrence of virus $\mathrm{C}$, cytomegalovirus infection and hypomagnesemia.

\section{Objective}

To estimate the incidence of new onset diabetes after liver transplantation and to identify factors associated with its development.

\section{Methods}

Retrospective cohort study of patients with liver transplantation between January 2011 and December 2013. Exclusion criteria: diagnosis of diabetes prior to transplantation, re-transplantation and combined transplantation.

\section{The diagnostic criteria of new onset diabetes post transplantation}

Fasting glycemia $\geq 126 \mathrm{mg} / \mathrm{dl}$ Blood glucose $\geq 200 \mathrm{mg} / \mathrm{dL}$ at two hours of oral glucose load test of $75 \mathrm{~g}$

$\mathrm{HbA} 1 \mathrm{c} \geq 6,5 \%$

Requirement for oral hypoglycemic agents or insulin to maintain normoglycemia.

\section{Classification of new onset diabetes post transplantation}

Early new onset diabetes: Diabetes diagnosis after transplant before the first year.

Transient diabetes: Diagnosis of diabetes after transplantation that reverts to the normal state before the first year..

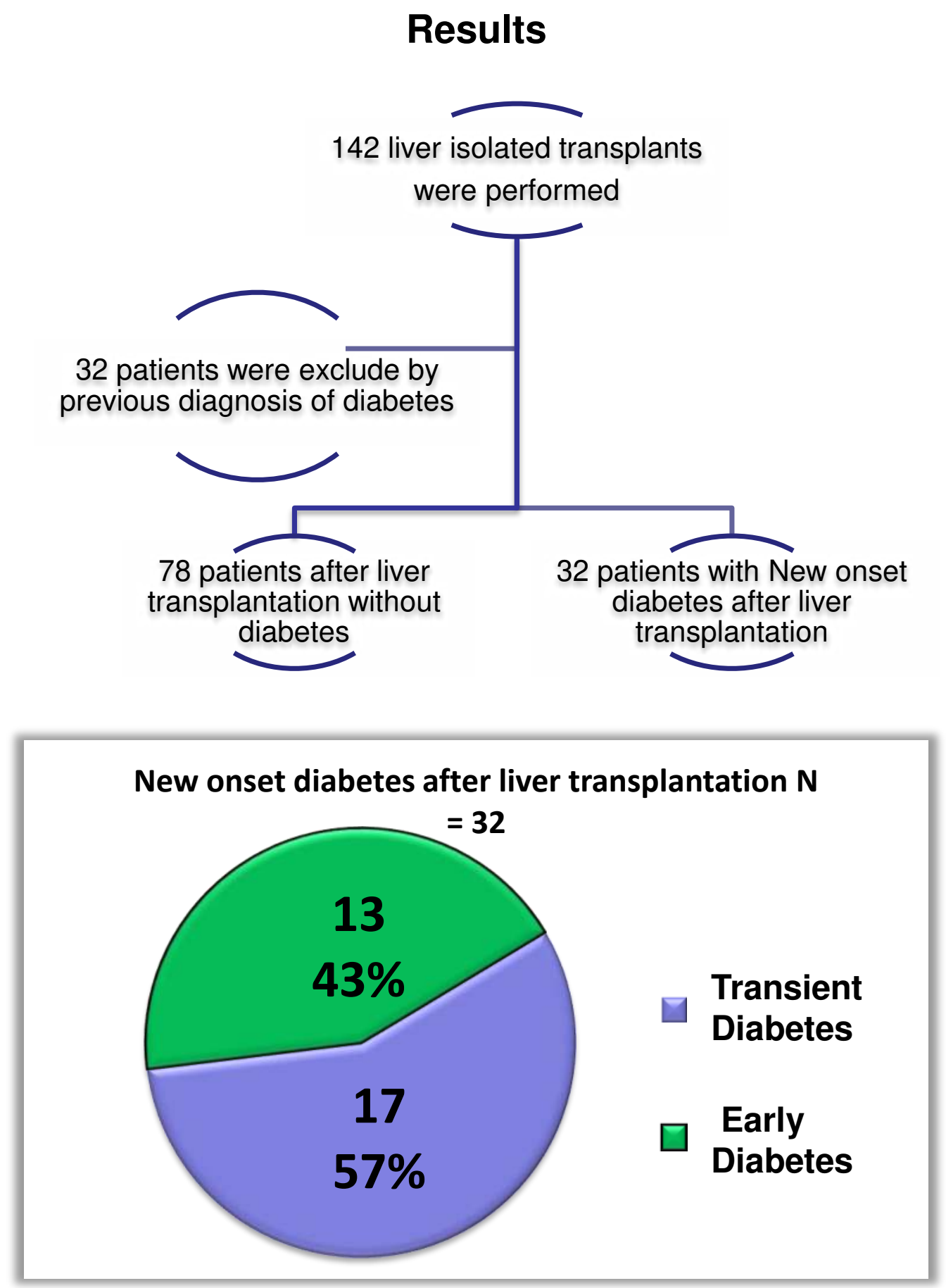

\begin{tabular}{|c|c|c|c|c|}
\hline Baseline Characteristics & $\begin{array}{l}\text { All patients } \\
\qquad N=110\end{array}$ & $\begin{array}{c}\text { No Diabetes } \\
N=78\end{array}$ & $\begin{array}{c}\text { Diabetes } \\
\mathrm{N}=32\end{array}$ & $\mathbf{P}$ \\
\hline Masculine Gender N / (\%) & $63(57 \%)$ & $39(50 \%)$ & $24(75 \%)$ & 0.016 \\
\hline Age (years) media $\pm \mathrm{DS}(\mathrm{N}=110)$ & $55.43 \pm 12.41$ & $\begin{array}{c}56.17 \pm \\
12.26\end{array}$ & $\begin{array}{l}53.625 \pm \\
12.79\end{array}$ & 0.3400 \\
\hline $\begin{array}{l}\text { Pre transplant hypertension } N / \% \\
(N=109)\end{array}$ & $13(11.93 \%)$ & $8(10.39 \%)$ & $5(15.63 \%)$ & 0.319 \\
\hline $\begin{array}{l}\text { Pre transplant dyslipidemia N/\% } \\
(\mathrm{N}=107)\end{array}$ & $14(13.08 \%)$ & $11(14.67 \%)$ & $3(9.38 \%)$ & 0.457 \\
\hline cirrhosis, $N / \%(N=110)$ & $99(90 \%)$ & $68(87.18 \%)$ & $31(96.88 \%)$ & 0.171 \\
\hline Score MELD $(\mathrm{N}=99)$ & $22.5(+-7.85)$ & $\begin{array}{l}23.08(+- \\
7.59)\end{array}$ & $\begin{array}{l}21.25(+- \\
8.37\end{array}$ & 0.30 \\
\hline $\begin{array}{l}\text { Calcineurin Inhibitors N./\% (N= } \\
\text { 105) }\end{array}$ & $102(97.14 \%)$ & $72(97.30 \%)$ & $30(96.77 \%)$ & 0.654 \\
\hline $\begin{array}{l}\text { Type of Calcineurine inhibitors } \\
\text { N./\% ( } \mathrm{N}=80) \\
\text { - Tacrolimus } \\
\text { - Cyclosporine }\end{array}$ & $\begin{array}{l}80(76.19 \%) \\
25(23.81 \%)\end{array}$ & $\begin{array}{l}55(74.32 \%) \\
19(25.68 \%)\end{array}$ & $\begin{array}{l}25(80.65 \%) \\
6(19.35 \%)\end{array}$ & 0.488 \\
\hline $\begin{array}{l}\text { Corticosteroids at } 6 \text { months. } N / \% \\
(N=101)\end{array}$ & $90(89.11 \%)$ & $62(8.57 \%)$ & $28(90.32 \%)$ & 0.794 \\
\hline $\begin{array}{l}\text { Corticosteroids at } 1 \text { year. } N / \% \\
(N=106)\end{array}$ & $75(70.75 \%)$ & $53(70.67 \%)$ & $22(70.97 \%)$ & 0.975 \\
\hline Mycophenolate $\mathrm{N} / \%(\mathrm{~N}=106)$ & $61(57.55 \%)$ & $42(56 \%)$ & $19(61.29 \%)$ & 0.616 \\
\hline Acute rejection $\mathrm{N} / \%(\mathrm{~N}=99)$ & $19(19.19)$ & $14(20.59)$ & $15(16.13)$ & 0.601 \\
\hline CMV infection $N / \%(N=110)$ & $35(31,82 \%)$ & $26(33,33 \%)$ & $9(28,13 \%)$ & 0.594 \\
\hline $\begin{array}{l}\text { Recurrence of hepatitis C N/\% } \\
(\mathrm{N}=109)\end{array}$ & $13(11.93 \%)$ & $5(6.49 \%)$ & $8(25 \%)$ & 0.011 \\
\hline $\begin{array}{l}\text { Hypomagnesemia pre transplant } \\
\mathrm{N} / \%(\mathrm{~N}=110)\end{array}$ & $32(29.9 \%)$ & $19(24.36 \%)$ & $13(40.63 \%)$ & 0.088 \\
\hline $\begin{array}{l}\text { Post-transplant } \\
\text { hypomagnesemia N/\% (N=87) }\end{array}$ & $79(90.80 \%)$ & 52 (91.23\%) & $27(90 \%)$ & 0.851 \\
\hline $\begin{array}{l}\text { Post-transplant HbA1c N/\% } \\
(\mathrm{N}=87)\end{array}$ & $5,18(1.01)$ & $5.17(1.14)$ & $5.18(0.79)$ & 0.995 \\
\hline $\begin{array}{l}\text { Post-transplant HbA1c N/\% } \\
(\mathrm{N}=93)\end{array}$ & $\begin{array}{l}103.65 \\
(28.68)\end{array}$ & $\begin{array}{l}101.58(+/- \\
32.1)\end{array}$ & $\begin{array}{l}107.8(+/- \\
20.0)\end{array}$ & 0.25 \\
\hline
\end{tabular}

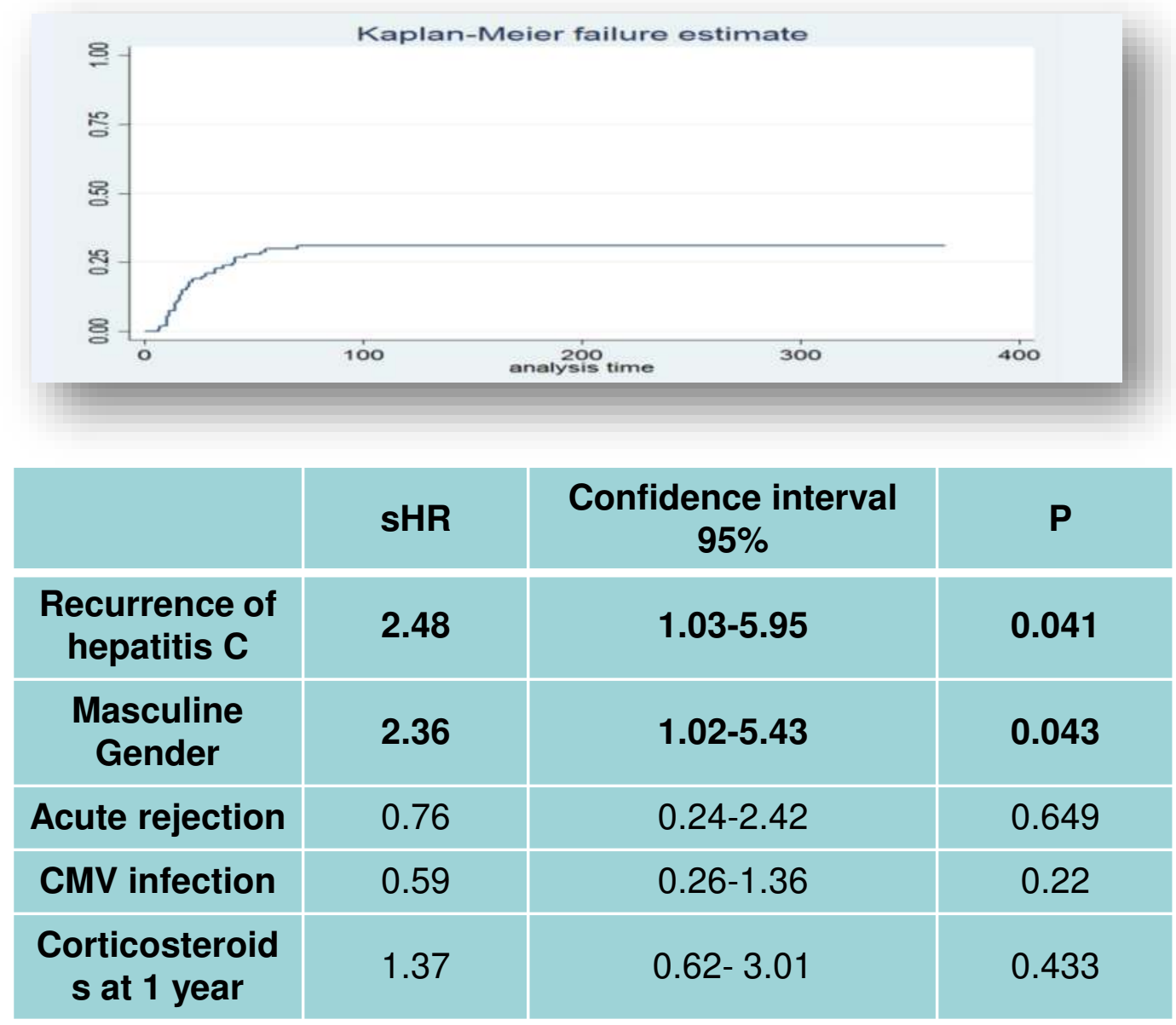

\section{Discussion}

We found a similar incidence of new onset diabetes after liver transplantation than in others reports. The only factor associated with the development of new onset diabetes post liver transplantation was recurrence of virus

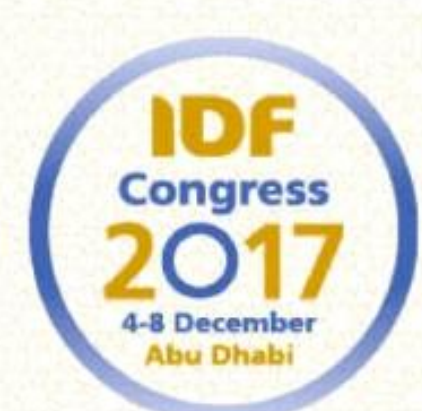

. Wallia A Illuri V, Molitch ME, Diabetes Care After Transplant: Definitions, Risk Factors, and Clinical Management. Med Clin North Am. 2016 May;100(3):535-50.

2. Report of the Expert Committee on the Diagnosis and Classification of Diabetes Mellitus. Diabetes Care, 1997. 20(7): p. 1183-97.

3. Davidson, J., et al., New-onset diabetes after transplantation: 2003 International consensus guidelines. Proceedings of an international expert panel meeting. Barcelona, Spain, 19 February 2003. Transplantation, 2003. 75(10 Suppl): p. SS3-24.

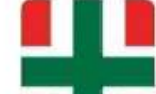

HOSPITAL ITALIANO

de Buenos Aires

javier.giunta@hiba.org.ar 